\title{
Attenuation Resilient AIF Estimation Based on Hierarchical Bayesian Modelling for First Pass Myocardial Perfusion MRI
}

\author{
Volker J. Schmid ${ }^{1}$, Peter D. Gatehouse ${ }^{2}$, and Guang-Zhong Yang ${ }^{1}$ \\ ${ }^{1}$ Institute for Biomedical Engineering, Imperial College, South Kensington, London, \\ United Kingdom \\ $\{$ v.schmid,g.z.yang\}@imperial.ac.uk \\ ${ }^{2}$ Cardiovascular Magnetic Resonance Unit, Royal Brompton Hospital, London, \\ United Kingdom \\ p.gatehouse@rbht.nhs.uk
}

\begin{abstract}
Non-linear attenuation of the Arterial Input Function (AIF) is a major problem in first-pass MR perfusion imaging due to the high concentration of the contrast agent in the blood pool. This paper presents a technique to reconstruct the true AIF using signal intensities in the myocardium and the attenuated AIF based on a Hierarchical Bayesian Model (HBM). With the proposed method, both the AIF and the response function are modeled as smoothed functions by using Bayesian penalty splines (P-Splines). The derived AIF is then used to estimate the impulse response of the myocardium based on deconvolution analysis. The proposed technique is validated both with simulated data using the MMID4 model and ten in vivo data sets for estimating myocardial perfusion reserve rates. The results demonstrate the ability of the proposed technique in accurately reconstructing the desired AIF for myocardial perfusion quantification. The method does not involve any MRI pulse sequence modification, and thus is expected to have wider clinical impact.
\end{abstract}

\section{Introduction}

Early diagnosis and localization of myocardial perfusion defect is an important step in the treatment of Coronary Artery Disease (CAD). For prognostic evaluation and monitoring the efficacy of interventional measures of patients with CAD, myocardial perfusion imaging plays an important role in establishing the ischaemic burden and the viability of ischaemic myocardium. In recent years, the development of myocardial perfusion cardiovascular MRI has extended its role in the evaluation of ischaemic heart disease beyond the situations where there have already been gross myocardial changes such as acute infarction or scarring [1]. The ability to non-invasively evaluate cardiac perfusion abnormalities before pathologic effects occur, or as follow-up to therapy, is important to the management of patients with CAD. Differentiation of ischaemic but viable myocardium from infarcted regions requires detailed global quantitative assessment and modeling of myocardial perfusion characteristics. In MRI, quantitative results have been achieved in animal studies with intravascular agents 

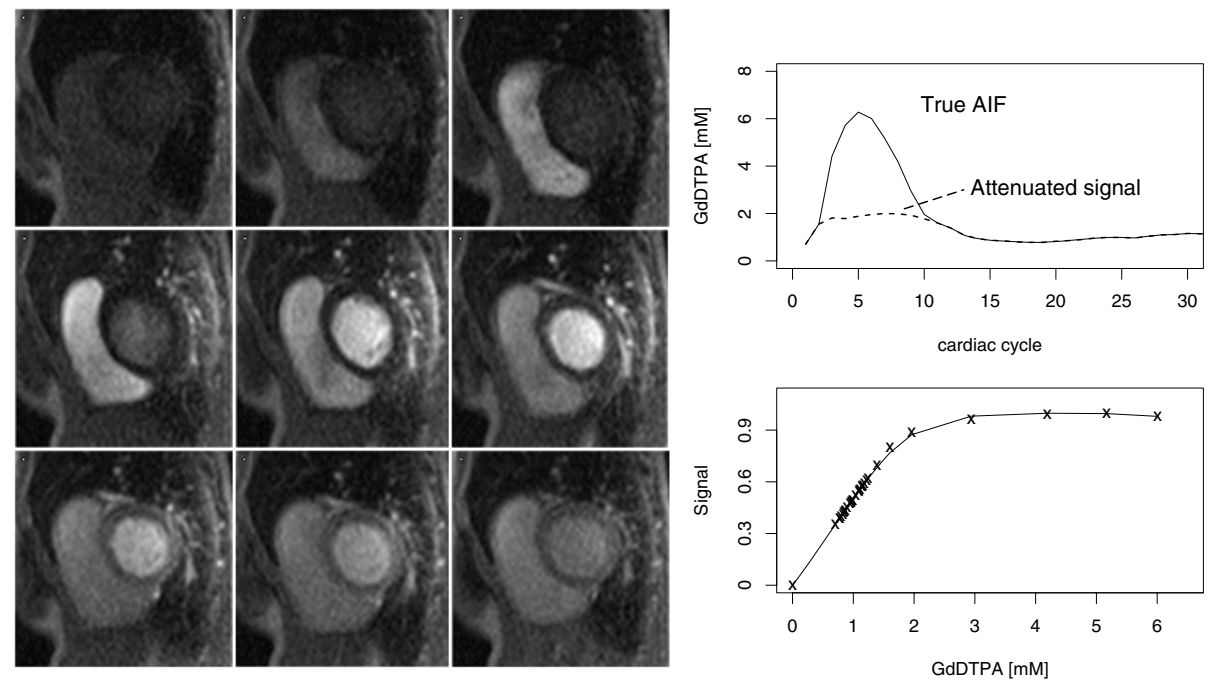

Fig. 1. Left: an example series of first pass myocardial perfusion MRI showing GdDTPA transit from the right ventricle, then to the left ventricle and finally entering into the myocardial tissue. Right top: attenuation of the AIF due to high Gd-DTPA concentration. Right bottom: Non-linear relationship between MR SI in the left ventricule (LV) and contrast concentration computed from a dual-imaging study [3].

(polylysine-Gd-DTPA) as a macromolecular blood pool marker and with conventional extracellular agents (Gd-DTPA) for human studies.

In general clinical practice, a high Gd-DTPA concentration is often required to achieve a good signal-to-noise ratio of the myocardium. However, the relationship between signal intensity (SI) and contrast concentration is linear only for low Gd-DTPA concentrations, typically up to about $2 \mathrm{mM}$ (for long saturation time delay) [2]. Beyond this value, the overall SI and contrast concentration are not linearly related, thus leading to a non-linear attenuation of the SI time curve as shown in Fig. 1, Although this is usually not a problem for myocardium, as the perfused Gd-DTPA is relatively low, the SI of the Arterial Input Function (AIF), usually measured in the Left-ventricular (LV) blood pool, can be severely attenuated [3]. This non-linear attenuation of the AIF can lead to large errors in the estimation of myocardial perfusion reserve derived from the impulse response of the Gd-DTPA bolus.

Extensive research in MRI pulse sequence design has been conducted recently to tackle the problem of non-linear attenuation. The dual-bolus technique [4, for example, uses a low gadolinium dose to measure the AIF, followed by a high dose for the myocardial tissue signal. However, this protocol is complicated to implement for routine clinical use and extensive care must be taken to ensure the reproducibility of the boluses. An alternative approach of using T1-fast acquisition relaxation mapping (T1-FARM) 5] computes the T1 signal from two gradient-echo $k$-space data sets, but the signal-to-noise ratio (SNR) for this 
method is low. To overcome this problem, a dual-imaging approach has been proposed [3], which uses a shorter inversion recovery time to measure the AIF and a longer recovery time to assess the myocardial perfusion response.

Considering the potential pitfalls of using these elaborated perfusion pulse sequences in routine clinical practices, we propose in this paper an attenuation resilient AIF estimation method based on conventional perfusion imaging protocols with high-dosage gadolinium boluses. A recent study [6] has demonstrated the value of a Bayesian technique for estimating a parametric AIF in dynamic-contrast enhanced MRI (DCE-MRI) using a parametric prior model of the AIF. In this paper, we propose a Hierarchical Bayesian Model (HBM) for the reconstruction of the AIF, where the measured AIF is estimated simultaneously with the response function. Both the AIF and the response function are modelled as smoothed functions by using Bayesian penalty splines (P-Splines) 7 . Since the information from the myocardium is sparse, a relatively informative prior model is used for the response function in the reconstruction step. Subsequently, the derived AIF can be used in existing myocardial impulse response estimation techniques based on deconvolution analysis. The proposed technique is validated both with simulated data based on the MMID4 model [8] and ten in vivo data sets for estimating $\mathrm{AIF}, \mathrm{MBF}$ and myocardial perfusion reserve (MPR) to demonstrate the potential clinical value of the technique.

\section{Theory and Methods}

Mathematically, HBM assumes the presence of latent variables, which are unknown and cannot be observed [9. A HBM typically consists of three stages. The data model defines how the observed data - in this study the SI measurements in the myocardial tissue $Y_{i t}$ and in the blood pool $Z_{t}$ - is generated from latent variables. The process model is a statistical description of the underlying physical process - in this study the flow of the contrast agent in blood and tissue, i.e. the (latent) response function in the myocardial tissue. In a third stage, prior distributions have to be specified to complete the HBM. Inference for the HBM is based on a Markov chain Monte Carlo (MCMC) algorithm.

\subsection{Data Model}

The observed signal intensities are realizations of stochastic processes, i.e. an observation consists of the signal and some noise. Let $S_{i}(t)$ be the true contrast concentration and $Y_{i t}$ be the observed signal intensity at time $t$ in section $i$ of the myocardium. Let $A(t)$ be the true AIF and $Z_{t}$ the observed signal intensity in the LV blood pool at time $t$. We assume that the signal intensity both in the myocardium and in the LV blood pool is the true intensity plus white noise $Y_{i t} \sim N\left(S_{i}(t), \sigma^{2}\right)$ for all $i, t ; Z_{t} \sim N\left(A(t), \rho_{t}^{2}\right)$ for all $t$. The variance of the observation error $\sigma^{2}$ is estimated from the data with a flat inverse Gamma prior $\sigma^{2} \sim I G\left(10^{-5}, 10^{-5}\right)$. The variance $\rho_{t}^{2}$, however, is a priori fixed in this study depending on $Z_{t}$. This is because we know $A(t)$ is equal to $Z_{t}$ for small values (up 
to observation error), so a small a priori variance of $\rho_{t}^{2}=10^{-5}$ is used. For higher values of $Z_{t}$ (i.e. $\left.Z_{t}>c \cdot \max \left(Z_{t}\right)\right)$ the $\mathrm{AIF}$ is attenuated. So a priori we assume a high variance $\left(e . g ., \rho_{t}^{2}=10 \cdot \max \left(Z_{t}\right)^{2}\right)$ for the AIF. So the prior information for the AIF is rather weak and the observed signal intensity in the myocardial tissue determines the AIF. The constant $c$ can be chosen subject-specific, usual values are between 0.4 and 0.5 .

\subsection{Process Model}

A general approach to modeling the blood flow in the myocardial tissue is to define the time curve of true signal in the myocardium $S_{i}(t)$ as a convolution of the arterial input function $A(t)$ and a response function $f$ such that

$$
S_{i}(t)=A(t) \otimes f_{i}(t)=\int_{0}^{t} A(t-u) f_{i}(u) d u .
$$

The signal intensity is measured at discrete time points $t_{1}, \ldots, t_{n}$, so Eqn. 1 can be discretized as

$$
\tilde{S}_{i t_{k}}=\sum_{l=1}^{T} A\left(t_{k}-t_{l}\right) f_{i}\left(t_{l}\right) \Delta t=\sum_{l=1}^{T} \tilde{A}_{k l} f\left(t_{l}\right),
$$

where $\Delta t$ represents the sampling interval [10]. The matrix $\tilde{\boldsymbol{A}}$ may be interpreted as a convolution operator and is defined via

$$
\tilde{A}_{k l}= \begin{cases}A\left(t_{k-l+1}\right) \Delta t & \text { for } k \leq l \\ 0 & \text { else. }\end{cases}
$$

Thus, the process model can be written as $\tilde{\boldsymbol{S}}_{i}=\tilde{\boldsymbol{A}} \boldsymbol{f}_{i}$ for all $i$.

\subsection{Prior Information}

We assume that both the response function and the AIF are smooth functions and can be approximated by B-Splines, i.e.

$$
f_{i}(t)=\sum_{j=1}^{p} \beta_{i j} B_{j t}, \quad A(t)=\sum_{j} \gamma_{j} B_{j t},
$$

where $\boldsymbol{B}$ is the $n \times p$ design matrix of $k$ th order B-splines with knots $s_{1}, \ldots, s_{p+k}$ [10. In vector notation, $\boldsymbol{f}_{\boldsymbol{i}}=\left(f_{i}\left(t_{1}\right), \ldots, f_{i}\left(t_{T}\right)\right)^{\prime}$ and Eqn. 4 may be expressed via $\boldsymbol{f}_{\boldsymbol{i}}=\boldsymbol{B} \boldsymbol{\beta}_{\boldsymbol{i}}, \tilde{\boldsymbol{A}}=\boldsymbol{B} \boldsymbol{\gamma}$, where $\boldsymbol{\beta}_{i}$ and $\boldsymbol{\gamma}$ are regression parameters. We plug this representation of $\boldsymbol{f}$ into $\tilde{\boldsymbol{S}}_{i}=\tilde{\boldsymbol{A}} \boldsymbol{f}_{i}$ and get

$$
\tilde{\boldsymbol{S}}_{i}=\tilde{\boldsymbol{A}} \boldsymbol{f}_{i}=\tilde{\boldsymbol{A}} \boldsymbol{B} \boldsymbol{\beta}_{i}=\boldsymbol{D} \boldsymbol{\beta}_{i},
$$

where $\boldsymbol{D}=\tilde{\boldsymbol{A}} \boldsymbol{B}$ is a $T \times p$ design matrix, which is the discrete convolution of the AIF with the B-Spline polynomials. However, in the HBM, $\boldsymbol{D}$ is dependent on $\boldsymbol{A}$, i.e. $\boldsymbol{D}$ is unknown and is estimated along with the other parameters. 
Following the theory of Bayesian P-Splines [7], a penalty function is used on the regression parameters $\boldsymbol{\beta}_{\boldsymbol{i}}$ and $\boldsymbol{\gamma}$, respectively. Here a second order difference is used as stochastic restriction, known as "random walk of second order", where

$$
\beta_{i t} \sim N\left(2 \beta_{i, t-1}-\beta_{i, t-2}, \phi_{i t}\right) \text { for } t>2, \text { for all } i
$$

and

$$
\gamma_{t} \sim N\left(2 \gamma_{t-1}-\gamma_{t-2}, \psi_{t}\right) \text { for } t>2 .
$$

Due to the fast upslope at the beginning of the contrast uptake compared to the rest of the perfusion sequence, an adaptive prior has to be used for smoothing. That is, the variance parameters $\phi$ and $\psi$ differ over time (and space) and are estimated from the data itself. To this end, flat inverse Gamma priors are used [7, where $\phi_{i t} \sim I G(a, b), \psi_{t} \sim I G(a, b)$ with $a=1, b=10^{-5}$.

\section{Simulation Study}

To evaluate the proposed HBM AIF estimation method, simulated myocardial perfusion intensity curves were generated using the MMID4 model [8]. Data was simulated to represent healthy subjects and patients with different grades of stenoses both in rest and under stress. For each group and state, 12 sections of the myocardium with different MBF values were simulated 10 times (values for healthy subjects are MBF at rest 0.8-1.0, MBF under stress 1.5-2.0, MPR 1.88-2.30; for subjects with stenosis: $\mathrm{MBF}$ at rest 0.8-1.0, MBF under stress 0.91.5, MPR 1.13-1.50). The simulated concentration series were then attenuated following the measurements of [2, i.e. translated into normalized T1-weighted signal intensities. Random noise with a SNR of 6:1 was added to the signal intensities. The attenuated AIF was then reconstructed with the proposed HBM algorithm. Fig. 2 (left) depicts the true AIF used in the simulation, showing the simulated attenuated SI time intensity curve in the LV and the 10 reconstructions of the AIF from data representing healthy patients under stress. In
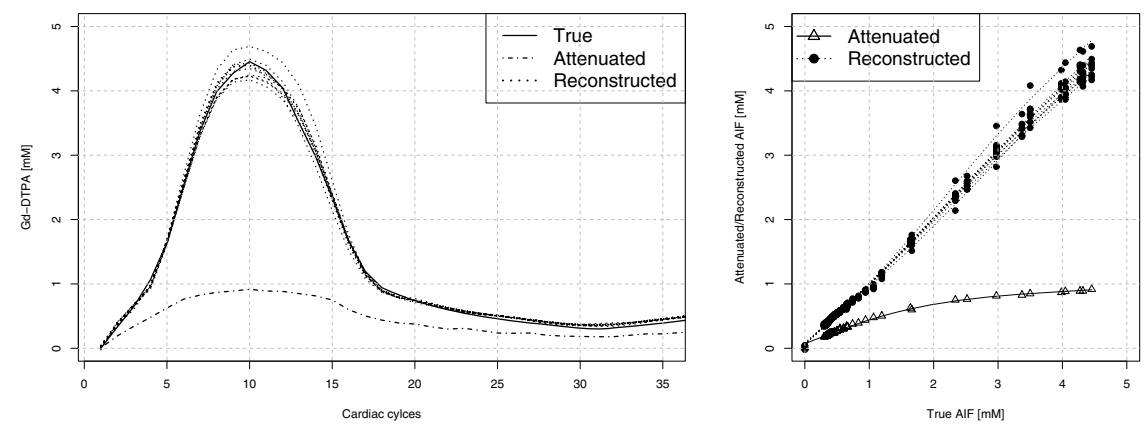

Fig. 2. Left: true AIF used in the simulation, attenuated AIF and the 10 reconstructions for a healthy patient under stress. Right: scatter plot of true AIF against attenuated and reconstructed AIF, respectively, with smoothing splines. 
Fig. 2 (right), the true AIF values are plotted against the attenuated AIF and the 10 reconstructions of the AIF. Cubic smoothing splines $(\lambda=1)$ are drawn for the reconstructions and the attenuated AIF, respectively.

The reconstructed AIF were then used in a semi-parametric analysis [1] to estimate the MBF at rest and under stress and the myocardial perfusion reserve (MPR), i.e. the ratio of hyperemic and baseline MBF. It can be deduced that with the reconstructed AIF, the mean overestimation of MBF is reduced from $118 \%$ to $28 \%$. The mean squared error of MPR estimation was reduced by $60.6 \%$ with the proposed reconstruction of the AIF. For the analysis with the attenuated AIF the $95 \%$ confidence intervalls (CI) of the estimated MPR values cover the true value in $33 \%$ of the sectors, whereas the $95 \%$ CI computed with reconstructed AIF always cover the true values.

\section{In Vivo Study}

To assess the clinical value of the proposed technique, the algorithm was applied to a study of 10 subjects. Results from the proposed technique were compared to results from a dual-imaging sequence [3. The latter uses scanning protocol which includes an additional FLASH sequence with short saturaiton-recovery time delay to directly measure the input function in the LV.

For this study [3, images were acquired with a $0.1 \mathrm{mmol} / \mathrm{kg}$ injection of a Gadolinium-based contrast agent on a 1.5-T Siemens Sonata scanner with singleshot FLASH with $48 \times 64$ resolution with a short saturation recovery time (SSRT) of $3.4 \mathrm{msec}, \mathrm{TE}=0.5 \mathrm{msec}, \mathrm{TR}=1 \mathrm{msec}$. This was followed by measurement in the same cardiac cycles with a $108 \times 256$ resolution on the same FOV with a longer saturation recovery time (LSRT) of $63.4 \mathrm{msec}$, TE $=1.2 \mathrm{msec}$, TR $=$ 1.86 msec. Each subject was scanned once under rest, followed by a scan after injection of $140 \mu \mathrm{g} / \mathrm{minute} / \mathrm{kg}$ of adenosine for four minutes, i.e. under stress.
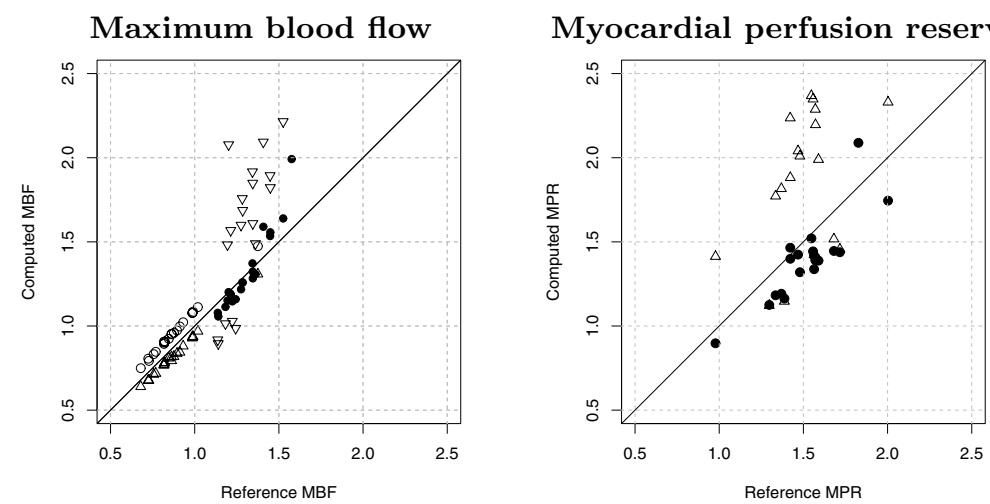

Fig. 3. Left: MBF computed with attenuated ( $\Delta$ at rest, $\nabla$ under stress) and reconstructed (o at rest, $\bullet$ under stress) AIF plotted against reference values from the dual-imaging technique. Right: MPR computed with attenuated $(\Delta)$ and reconstructed $(\bullet)$ AIF against reference values from the dual-imaging technique. 
Table 1. Mean squared difference between reference method and attenuated and reconstructed AIF for MBF at rest and under stress and MPR

\begin{tabular}{|c|c|c|c|c|c|c|c|c|c|c|}
\hline & \multicolumn{2}{|c|}{ Subject 1} & \multicolumn{2}{|c|}{ Subject 2} & \multicolumn{2}{|c|}{ Subject 3} & \multicolumn{2}{|c|}{ Subject 4} & \multicolumn{2}{|c|}{ Subject 5} \\
\hline & att. & rec. & att. & rec. & att. & rec. & att & rec. & att. & rec. \\
\hline$\overline{M B F}$ at rest & 0.886 & 0.407 & 80.039 & 1.680 & 3.640 & 1.581 & 2.937 & 1.484 & 1.078 & 1.570 \\
\hline MBF un & 1.658 & 0.945 & 1.269 & 0.281 & 2.489 & 0.678 & 3.096 & 0.929 & 1.163 & 0.962 \\
\hline \multirow[t]{3}{*}{ MPR } & 1.878 & 0.827 & 0.141 & 0.122 & 0.684 & 0.450 & 1.053 & 0.627 & 1.093 & 0.613 \\
\hline & \multicolumn{2}{|c|}{ Subject 6} & \multicolumn{2}{|c|}{ Subject 7} & \multicolumn{2}{|c|}{ Subject 8} & \multicolumn{2}{|c|}{ Subject 9} & \multicolumn{2}{|c|}{ Subject 10} \\
\hline & att. & rec. & att. & rec. & att. & rec. & att. & rec. & att. & rec. \\
\hline $\mathrm{MBF}$ at & 3.372 & 2.057 & 0.986 & 1.225 & 32.037 & 4.605 & 1.131 & 0.663 & 3.127 & 1.100 \\
\hline MBF ur & 3.356 & 0.629 & 1.195 & 0.944 & 0.937 & 0.033 & 0.864 & 0.438 & 2.614 & 1.035 \\
\hline MPR & 1.029 & 0.306 & 1.206 & 0.771 & 0.029 & 0.007 & 0.765 & 0.659 & 0.836 & 0.941 \\
\hline
\end{tabular}

Results from the analysis using three different input functions are used to illustrate the accuracy of the proposed technique:

a) the signal intensity measured in the LSRT scans, i.e. a conventional attenuated AIF,

b) the signal intensity measured in the SSRT scans, i.e. an AIF from the dualimaging technique, which is used as reference model here,

c) the AIF reconstructed with the proposed technique.

Maximum blood flow (MBF) and myocardial perfusion reserve (MPR) were computed from the data with the corresponding AIF. Fig. 3 (left) depicts the $\mathrm{MBF}$ at rest and under stress in the sectors of the myocardial tissue for subject 1. For the baseline scan, all three models agree reasonably well, i.e. non-linear attenuation does not represent a significant issue. However, for the hyperemic scan, MBF computed with the attenuated AIF is underestimated for small values and overestimated for larger values of MBF. MBF values computed from the reconstructed AIF however mostly agree with the reference method based on the dual-imaging sequence. Fig. 3 (right) depicts the MPR in different sections of the myocardium for subject 1 . MPR estimates with the attenuated AIF show large differences compared to the reference method, whereas results from the reconstructed method correspond well with the reference data.

Tab.1 lists the mean squared difference of MBF and MPR between reference method and attenuated and reconstructed estimates, respectively, for the 10 subjects studied. The mean squared difference is noticeably reduced for all subjects.

\section{Conclusion}

In this paper, we have presented a technique for dealing with attenuated AIF in myocardial perfusion imaging based on HBM. The HBM uses information from the observed attenuated signal in the LV blood pool along with information from the signal in the myocardial tissue based on a smoothness constraint and Bayesian 
P-splines [7]. Compared to recently proposed techniques such as dual-imaging, dual-bolus or T1-FARM, the proposed technique does not involve any MRI pulse sequence modification, and thus is more practical for routine clinical use. Although the proposed technique assumes a smooth AIF, the algorithm does allows for rapid changes. The cutoff parameter $c$ has to be choosen patient-specifically; for all subjects in the in vivo study values of $c$ between 0.4 and 0.5 were sufficient and changes to the $c$ parameter of \pm 0.1 did not change the results significantly.

Application of the proposed technique to simulated data and to in vivo scans clearly demonstrate the ability of the proposed technique to accurately reconstruct the desired AIF for myocardial perfusion quantification. The results illustrate the problems of correct estimation of MBF and MPR when using an attenuated AIF; especially for higher values of MBF (e.g., for healthy patients under stress). By using the reconstructed AIF with HBM, estimates of MBF and MPR are similar to the ground truth data derived from the reference scans.

\section{References}

1. Panting, J., Gatehouse, P., Yang, G.-Z., Grothues, F., Firmin, D., Collins, P., Pennell, D.: Abnormal subendocardial perfusion in cardiac syndrome $\mathrm{X}$ detected by cardiovascular MRI. New Engl. J. of Med. 346, 1948-1953 (2002)

2. Kim, D., Axel, L.: Multislice, dual-imaging sequence for increasing the dynamic range of the contrast-enhanced blood signal and CNR of myocardial enhancement at 3T. J. of Mag. Res. Imag. 23, 81-86 (2006)

3. Gatehouse, P., Elkington, A., Ablitt, N., Yang, G., Pennell, D., Firmin, D.: Accurate assesment of the arterial input function during high-dose myocardial perfusion cardiovascular magnetic resonance. J. Mag. Res. Imag. 20, 39-45 (2004)

4. Christian, T.F., Rettmann, D.W., Aletras, A.H., Liao, S.L., Taylor, J.L., Balaban, R.S., Arai, A.E.: Absolute myocardial perfusion in canines measured by using dualbolus first-pass MR imaging. Radiology 232, 677-684 (2004)

5. Bellamy, D.D., Pereira, R.S., McKenzie, C.A., Prato, F.S., Drost, D.J., Sykes, J., Wisenberg, G.: Gd-DTPA bolus tracking in the myocardium using T1 fast acquisition relaxation mapping (T1 FARM). Magn. Res. in Med. 46, 555-564 (2001)

6. Orton, M.R., Walker-Samuel, S., Collins, D.J., Leach, M.O.: A joint bayesian method for robust estimation of PK and AIF parameters for DCE-MR imaging. In: Proceedings of the 14th Annual Meeting of ISMRM, Seattle, p. 3490 (2006)

7. Lang, S., Brezger, A.: Bayesian P-splines. J. of Comp. and Graph Stat. 13, 183-212 (2004)

8. Kroll, K., Wilke, N., Jerosch-Herold, M., Wang, Y., Zhang, Y., Bache, R.J., Gassingthwaighte, J.B.: Modeling regional myocardial flows from residue functions of an intravascular indicator. Am. J. of Heart. Circ. Phys. 271, 1643-1655 (1996)

9. Gilks, W.R., Richardson, S., Spiegelhalter, D.J.: Markov Chain Monte Carlo in Practice. Chapman \& Hall, London (1996)

10. Jerosch-Herold, M., Swingen, C., Seethamraju, R.: Myocardial blood flow quantification with MRI by model-independent deconvolution. Med. Phys. 29(5), 886-897 (2002)

11. Schmid, V.J, Whitcher, B., Yang, G.Z.: Semi-parametric analysis of dynamic contrast-enhanced MRI using Bayesian P-splines. In: Larsen, R., Nielsen, M., Sporring, J. (eds.) MICCAI 2006. LNCS, vol. 4190, pp. 679-686. Springer, Heidelberg (2006) 